\title{
Significados del concepto medio ambiente en profesores que enseñan geografía en contextos rurales de Chile ${ }^{1}$
}

\author{
Andoni Arenas Martija ${ }^{2}$ y Patricio Pérez Gallardo³
}

\begin{abstract}
RESUMEN
En la Educación Básica chilena los contenidos de Geografía más relevantes son ubicación espacial, identificación de elementos del Paisaje, sistema de referencias cartográficas y Medio Ambiente. Este concepto es el de mayor presencia en el currículo, careciendo de una clara conceptualización y desposeído de relaciones con otros conceptos geográficos. Se presenta en este texto una aproximación al concepto Medio Ambiente basada en los significados que dan los/as profesores/as, indagando en las relaciones entre Medio Ambiente y contenidos de la Geografía Escolar. Esto, en el marco de una investigación interpretativa con una muestra intencionada de 45 profesores/as rurales de 3 regiones de Chile, realizando entrevistas semiestructuradas, Análisis de Contenido a los datos y Triangulación Teórica. Los resultados muestran tres significados de Medio Ambiente: ligado al cuidado-reciclaje-limpieza, sinónimo de entorno y vinculado a la preocupación por la degradación. Finalmente, algunos profesores/as relacionan Medio Ambiente con problemáticas que debe enseñar la Geografía Escolar.
\end{abstract}

Palabras clave: Medio ambiente, significados, profesores de geografía, naturaleza, entorno.

\begin{abstract}
In the Chilean Basic Education, the most relevant Geography contents are spatial location, identification of elements of the Landscape, system of cartographic references and Environment. This concept has the greatest presence in the curriculum, lacking a clear conceptualization and dispossessed of relationships with other geographic concepts. An approximation to the concept of the Environment is presented in this text, based on the meanings given by the professors, investigating the relationship between the Environment and the contents of the School Geography. This, in the framework of an interpretative research with an intentional sample of 45 rural teachers from 3 regions of Chile, conducting semi-structured interviews, Data Content Analysis and Theoretical Triangulation. The results show three meanings of Environment: linked to care-recycling-cleaning, synonym of environment and linked to the concern for deterioration. Finally some professors relate Environment with problems that School Geography should teach.
\end{abstract}

Keywords: Environment, Meanings, Teachers of Geography, Nature, Surroundings 
La problemática que aborda este artículo tiene como contexto la debilidad conceptual y el descuido temático del currículo chileno en el ámbito geográfico (Arenas et al., 2016), específicamente en uno de sus conceptos claves: Medio Ambiente (Garrido, 2005). Este concepto aparece numerosas veces en los textos curriculares, junto a Territorio, Paisaje y Región, con claras debilidades teóricas y sin proveer el desarrollo de aprendizajes complejos en los ámbitos cognitivos y de sus habilidades asociadas. La impronta curricular está en proteger y cuidar como expresión de valor del concepto de Medio Ambiente, sin saber a cabalidad qué se entiende o qué se podría entender por tal concepto. Un ejemplo de esto, es lo que pasa en el Módulo de Educación Rural, donde el concepto no se encuentra definido (MINEDUC, 2014).

Lo anterior es relevante por la diferencia que se produce entre el bajo peso que tiene la conceptualización geográfica en el sistema educativo chileno con el gran desarrollo que ha tenido la enseñanza por conceptos en la Educación Geográfica (Cavalcanti, 2009). Hay algunas propuestas alternativas de diferente alcance, impacto y consistencia que han equilibrado estos elementos como por ejemplo, la Educación Ambiental que se organiza en torno a conceptos e ideas científicas, desarrollo de valores y despliegue de habilidades complejas, en síntesis, con una propuesta didáctica específica (Saribas et al., 2016). Diversos estudios evidencian la importancia de la enseñanza de estas temáticas y los aportes que desde la Geografía se pueden desarrollar para la comprensión del concepto Medio Ambiente (Gurevich, 2011; Ângelo, 2011; Cardoso, 2013). No obstante, Leff (2008) plantea que, si bien las temáticas medio ambientales son de gran importancia para racionalizar la complejidad de la crisis ambiental, aún no logran salir de la periferia del sistema educativo.

Esta debilidad conceptual se acentúa en la deficiente formación inicial y permanente de los/ as profesores/as en lo geográfico y en su didáctica escolar, lo que refuerza que el currículo oficial pase a ser, prácticamente, la única guía temática y conceptual que orienta lo enseñado (Arenas et. al, 2016). Sin embargo, en determinados casos, las experiencias espaciales-geográficas, tanto personales como profesionales, ampliadas y llevadas al aula creativamente, suplen en parte la precariedad conceptual, tanto del currículo, como de la formación inicial y la formación permanente (Arenas et al., 2017). Es presumible que los profesores/as poseen un manejo conceptual débil y que los significados atribuidos a Medio Ambiente estén mediados por otras fuentes de conocimiento.

La investigación que sirvió de marco a la indagación particular presentada aquí, tenía por objetivo “identificar el significado de lo Geográfico en las prácticas de enseñanza de los docentes de la Red Maestros de Maestros y de Microcentros Rurales" ${ }^{\prime 4}$ y que se profundizó en los significados que le dan estos/as profesores/as al concepto de Medio Ambiente, contrastándolos con el currículo nacional y las caracterísitcas de la formación inicial/permanente de estos/as profesores/as. Específicamente se quería dilucidar cuáles eran los principales rasgos de estos significados sobre Medio Ambiente, como, por ejemplo, sus compomentes o elementos presentes en la conceptualización así como ver sí las temáticas medioambientales estaban más relacionadas con la Geografía y/o las Ciencias Naturales.

Fondecyt 11130400. 2013-2015. ¿Qué Geografía enseñamos? El Significado de lo Geográfico en las prácticas de enseñanza de los docentes de la Red Maestros de Maestros y de Microcentros Rurales. 


\title{
Marco teórico referencial: Medio Ambiente como concepto y su relación con la Geografía
}

\author{
Medio Ambiente como concepto
}

El concepto Medio Ambiente en las últimas décadas ha adquirido una difusión masiva que, si bien resulta beneficiosa para aproximarse a las problemáticas ambientales actuales, no está exenta de banalizaciones producto de malas, descontextualizadas y tergiversadas interpretaciones del concepto (Gallastegui y Galea, 2009). Desde la Geografía, el concepto ha permitido vincular la realidad natural con la realidad social en un determinado espacio geográfico. Según Moreira (2012) el concepto aborda lo natural y lo social como determinaciones de la existencia, las cuales acaban relacionándose en el proceso de reproducción de las dinámicas humanas, en las cuales se desarrolla una socialización de la naturaleza y una naturalización de la sociedad. En ese sentido, el proceso de producción de espacio sería producción de naturaleza y, por ende, ese todo procesual sería el Medio Ambiente (Moreira, 2012). Por lo tanto, el concepto Medio Ambiente acaba refiriéndose a las relaciones existentes en el espacio geográfico -al igual que Territorio, Lugar o Paisaje-, pero priorizando su atención exclusivamente en el diálogo existente entre los elementos naturales y sociales. En tal sentido, el concepto acaba siendo una más de las categorías analíticas de Espacio Geográfico.

Con la finalidad de comprender de mejor modo el vínculo existente entre lo humano y lo natural en el Espacio Geográfico, por ende, la singularidad y especificidad del concepto Medio Ambiente, se hace necesario explicitar nuestra concepción del propio Espacio Geográfico. Según Santos (2004) el espacio geográfico es explicado como un conjunto indisociable de sistema de objetos y sistema de acciones, resultando ser un hecho social producto de las relaciones históricas entre los individuos y los grupos humanos. Una noción de espacio que se vincula con ésto es la construida por Massey (2004), quien se refiere al espacio en su condición de "relacional"; en su condición de "múltiple", por los múltiples actores que lo configuran, sus diversas identidades y prácticas espaciales; y en su condición "transformacional", tanto por los cambios a escala local como global. Al respecto, Soja afirma que las personas en su condición de seres intrínsecamente espaciales están "comprometidos en la actividad colectiva de producción de espacios" (Soja, 2008: 33), donde nuestros pensamientos y acciones modelan los espacios en los que vivimos, a la vez que tales espacios y lugares producidos colectivamente modelan nuestros pensamientos y acciones.

Sí se asume el concepto Medio Ambiente como las relaciones humano-naturaleza del Espacio Geográfico, se vuelve imprescindible detenernos en las conceptualizaciones de naturaleza y técnica, puesto que para Santos (2004), el Medio Ambiente puede ser entendido/estudiado en base a la mayor o menor carga de ciencia, tecnología e información que tengan las dinámicas de determinado Espacio Geográfico, existiendo casos en los cuales lo artificial se sobrepone a la naturaleza y la substituye.

Naturaleza se vuelve un concepto relevante para lograr comprender las diferencias y relaciones entre lo natural y lo cultural. Según Van Dooren (2014) si nos esforzásemos por diferenciar ambos conceptos asignando como natural todo aquello no-humano, en pleno siglo XXI nos en- 
frentaríamos a la realidad de que ya no hay naturaleza (Van Dooren, 2014; Hinchliffe, 2005). Para Castoriadis (2013), si bien el límite entre lo humano y lo natural es difícil de demarcar, hay un punto de división, el cual radica en la existencia de lo estrictamente natural, con leyes y reglas propias, previas a cualquier intervención humana, lo que constituye el "primer estrato natural", demarcando un límite con lo antrópico en cuanto sus dinámicas originarias no corresponden a alteraciones humanas.

El primer estrato natural solo ha podido ser modificado e intervenido por el humano mediante la utilización de la técnica (Santos, 2004; Porto Gonçalves, 2001), la que en distintos momentos de la historia ha mediado la relación humano-naturaleza. Así ha sido, por ejemplo, con el establecimiento de la agricultura como práctica de abastecimiento alimenticio o con la conformación de ciudades y la organización socio-territorial en Estados, como también con la invención de barcos, ferrocarriles y aviones, inventos que fueron dibujando y rediseñando el mapa geopolítico universal durante los últimos siglos. Las últimas décadas han presenciado como novedad la creación de la ingeniería genética y los avances en biotecnología (Moreira, 2012), los cuales han reestructurado la explotación de "recursos naturales", reconfigurando además de las dinámicas urbano-rurales, las lógicas de extractivismo global (Alimonda, 2011; Gudynas, 2009), y consigo la relación de poder entre los humanos a diferentes escalas, entre éstos y la naturaleza.

Si bien el "primer estrato natural" logra diferenciar lo natural de lo social, lo hace sin omitir los vínculos existentes entre ambos aspectos de la vida en el planeta. La naturalización de la sociedad y la socialización de la naturaleza a la que Moreira (2012) hacía referencia, está presente en casi todos los aspectos de nuestra realidad, y aquellas relaciones no pueden ser desconocidos para comprender la espacialidad geográfica (Hinchliffe, 2005). Es entonces que entran en conflicto las concepciones que posicionan a la naturaleza como un espacio virgen externo a la sociedad con aquellas que reconocen los diferentes grados de artificialidad de la naturaleza en relación a las intervenciones tecnológicas que el humano ha desarrollado en ella (Gregory et al., 2009) e incluso aquellas que incorporan al humano y sus variadas intervenciones como parte de otra naturaleza, la cual lo incluye. Vale decir también, que las ilaciones entre ambas esferas no se agotan solo en la utilización de la técnica o en visiones representativas de la Geografía, pues hay autores que desde la Geografía Cultural logran comprender a la naturaleza como una fuente de emociones (Little, 2008), reconociendo en relación a ella sensaciones de miedo o protección en los humanos e incluso identificando diferencias en los modos de habitarla según su contexto rural o urbano. Entonces las relaciones entre naturaleza y cultura quedan abierta a un abanico de posibilidades imaginables, cuyo foco de estudio es parte de los estudios ambientales.

\section{Medio Ambiente y Geografía}

Sin embargo, es importante mencionar la omisión del carácter humano como un patrón más o menos permanente en el abordaje teórico del concepto Medio Ambiente, así como en las investigaciones ambientales y en la enseñanza escolar de la Geografía. Desde el ámbito propiamente geográfico, en la década de 1970 Gourou (1973) señalaba que tal omisión era producto de que el estudio del medio humano requería la integración de aspectos no visibles en el Paisaje, tales como la técnica, la historia y otros factores civilizatorios que han forjado los elementos humanos visibles: asentamientos, infraestructura, explotación de recursos naturales. Por defecto, el estudio del Medio Ambiente enfatizó en el análisis del medio natural, como si solo existiera ese primer estrato 
poseedor de relaciones monocausales con el estrato humano (Castoriadis, 2013). Este aspecto, es herencia de una Geografía que concebía el Espacio Geográfico como contenedor de elementos físicos, escenario de las acciones humanas y no como construcción-producción social. Esta contradicción epistemológica ha tenido profundas huellas es la Geografía Escolar, específicamente en la comprensión de la interacción humano-naturaleza, evidenciándose la pugna entre las "antiguas" y "nuevas" concepciones de Medio Ambiente y de Geografía (Arenas y Salinas, 2013).

Por el contrario, comprendemos Medio Ambiente como una categoría analítica del Espacio Geográfico, en la cual se da énfasis a las relaciones entre humanos y naturales, lo que permite prestar atención y buscar soluciones a las cuestiones ambientales-sociales-territoriales. En ese sentido, el concepto proporciona una plataforma teórica para analizar los actuales conflictos socio-ambientales, facilitando el diálogo de la Geografía con otras ciencias y con los problemas reales de la sociedad en base al nexo del Medio Ambiente con conceptos como Derechos Humanos, Derecho Indígena, Sociedad Civil, Ciudadanía, Autonomía y Resistencias, entre otros posibles (Araya et al., 2013; Callai y De Moraes, 2016).

Vale decir, que la importancia de instalar la temática ambiental en la educación formal radica en la urgente necesidad de asumir y abordar la complejidad de la crisis ambiental actual y al igual que Haraway (2015) no nos referimos solo al cambio climático, más bien, hablamos del conjunto de conflictos ambientales asociados, por ejemplo, a la liberación de productos químicos tóxicos, a los impactos de la minería, a la contaminación de aguas superficiales y subterráneas, a las diversas problemáticas de las metrópolis o al uso de bienes tan fundamentales como el agua y el suelo.

Lo anterior se da en el contexto mundial de una "tercera revolución industrial" caracterizada por la ingeniería genética y la financiarización como nuevo sistema de acumulación (Moreira, 2012), y por la instalación de lógicas neoliberales de desarrollo (Smith, 2005) que repercuten en el control económico, político y cultural de los espacios, sus bienes y sus usos. Produciendo un desarrollo territorial desigual de los mismos a escala mundial-local (Harvey, 2007; McDowell, 2000) donde las regiones subdesarrolladas han implementado modelos ligados al extractivismo con resultados devastadores para muchos ecosistemas y poblaciones humanas (Alimonda, 2011; Porto Gonçalves, 2001).

En ese sentido, la cuestión ambiental debe ir más allá, no centrándose solo en "innovar tecnologías para reciclar los desechos contaminantes" o "incorporar normas ecológicas a los agentes económicos" (Leff, 2007: 52), sino que debe poner en valor la diversidad cultural y étnica, fomentando desde ahí las distintas formas de manejo productivo en armonía con la naturaleza. Se abandona entonces, la reducción que se ha hecho de la discusión ambiental a aspectos técnicos (Gudynas, 2002), que ha convertido el trabajo medioambiental en un simple "gerenciamiento" del cuidado de la naturaleza, para abordar las temáticas ambientales desde su complejidad. Al considerar estos desafíos se hace necesario diseñar nuevos procesos formativos para los/as profesores/as, que impliquen mejores herramientas de trabajo y una re-lectura de su contexto (Bianchi et al., 2017) puesto que se ha constatado la debilidad del conocimiento disciplinar, y especialmente conceptual, de los/as profesores/as que enseñan geografía en el sistema escolar (Arenas et al., 2016).

Con todo, los significados que los/as profesores/as le dan a la Geografía en general y a Medio Ambiente en particular, no se explica únicamente desde su formación inicial/permanente ni tampoco puede ser entendidos como el grado de conocimiento formal que se tiene. Desde los 
planteamientos del constructivismo social de la realidad (Berger y Luckmann, 1996) como de su espacialidad, que es lo específicamente geográfico (Nogué, 2007; Nogué y Romero, 2006) se comprende que los fenómenos humanos, entre ellos los educativos y geográficos, se desarrollan en contextos sociales particulares mediante construcciones (conceptos-prácticas) que son resultado de negociaciones de orden social- espacial (Schwandt, 1998). De esta forma los significados son elaborados por los/as docentes que enseñan un contenido que "saben" y que a la vez "viven" (Tuan, 2007; Garrido, 2005), por lo tanto, portan un significado de lo geográfico que ponen en juego al enseñar.

\section{Diseño Metodológico}

El diseño general de la investigación tuvo un carácter interpretativo-exploratorio e implementado en secuencias de recopilación y análisis de datos siguiendo los planteamientos generales de (Denzin y Lincoln (2005). La muestra intencionada (Flick, 2014) estuvo compuesta por 45 profesores y profesoras que enseñanza Geografía entre primer y sexto año de primaria, miembros de los Microcentro Rurales. Estos Microcentros pertenecían a 7 municipios diferentes, localizados en 3 Regiones de Chile (Coquimbo, IV Región; Puchuncaví, La Calera, Hijuelas, Villa Alemana, Quilpué, V Región; Tomé, VIII Región). Los Microcentros agrupan a profesores que trabajan en escuelas en amplias zonas rurales ${ }^{5}$, es decir, aisladas o con menor accesibilidad (Avalos, 1999), implementándose desde 1992 como una estrategia para mejorar las prácticas pedagógicas en el aula a través de reuniones periódicas de trabajo entre los docente que los componen de acuerdo a un programa de trabajo preestablecido y con apoyo de los supervisores del MINEDUC ${ }^{6}$.

A estos 45 profesores/as se les realizó una entrevista semiestructurada en profundidad de aproximadamente una hora y media (Longhurst, 2010). A los datos recogidos se les aplicó Análisis del Contenido en base a unidades analíticas (Gibbs, 2012). Las entrevistas se realizaron y transcribiero durante el segundo semestre del año 2014, el Análisis del Contenido general se desarrolló durante el año 2015 y la especificidad relativa al concepto de Medio Ambiente, durante el primer semestre de 2016, a través de Triangulación Teórica (Flick, 2004).

Los resultados fueron organizados en cuatro grupos, los tres primeros exponen tres significados distintos que los/as profesores/as le dan al concepto Medio Ambiente. En cambio, el cuarto, expone la relación que los/as profesores/as establecen entre el concepto Medio Ambiente y la Educación Geográfica en la Escuela.

\section{Medio Ambiente como cuidado, reciclaje, y limpieza}

Una de las nociones de medio ambiente presentes en las respuestas dadas por profesores/as, es la que vincula tal concepto con las ideas de "cuidado, reciclaje y limpieza". La significación del concepto Medio Ambiente desde la perspectiva del cuidado ha tratado al concepto como sinónimo del concepto "naturaleza", donde la idea central está focalizada en "proteger" la naturaleza

Datos disponibles en http://www.redinnovemos.org/content/view/662/81/lang,sp/

Disponible en http://centroestudios. mineduc.cl/index.php?t=96\&i=2\&cc=2044\&tm=2 
de la acción humana, es decir, lo que los/as profesores/as mencionan como "cuidado del medio ambiente":

"Más que nada el concepto de medio ambiente, el cuidado y la contaminación que se hace con el medio ambiente, en ese contexto se ha trabajado ¿ya?, recientemente, como se contamina, como contaminan ellos [refiriéndose a los estudiantes], en que forma, de hecho, se han hecho campañas respecto a eso, el cuidado del medio ambiente, (...)". Profesor/a 1.

“(...) uno tiene que referirse a cómo cuidar el medio ambiente, en diferentes hábitats por ejemplo (...)". Profesor/a 7.

En ambos testimonios se reconoce el trato sinonímico de los conceptos Medio Ambiente y naturaleza, donde el foco de análisis está puesto en el cuidado y la protección de la naturaleza, y no en la comprensión de medio ambiente como el conjunto de relaciones establecidas entre el sistema humano y el sistema natural en un espacio geográfico determinado.

Otra significación dada a Medio Ambiente, muy ligada a la de "cuidado" es la que relaciona tal concepto con la idea de "reciclaje", en la cual el concepto queda reducido a la clasificación y reutilización de los desechos producidos por el humano; de este modo, hubo un grupo de profesores/as que al responder qué comprenden o cómo trabajan el concepto de Medio Ambiente afirmaron lo siguiente:

"Se hizo campaña de reciclaje de papel el año pasado, separamos los tipos de papel con todos los niños (...), también hicimos una campaña de tratamiento de botellas plásticas (...)". Profesor/a 47.

“(...) lo hemos tratado de hacer, incluso tratamos de separar por basurero plástico y cosas, no funcionó. Lo que pasa es que aquí los niños llegan y hacen, como hay muchos que les cuesta leer o aprender, no separan por basurero, incluso se ocupan cosas y el tema de medio ambiente aquí no es muy agradable". Profesor/a 11.

Una tercera significación dentro de este grupo es la que enlaza el concepto Medio Ambiente con la "limpieza" del lugar que se habita. Muchos profesores/as al momento de responder si habían trabajado o no el concepto Medio Ambiente enfatizan en temas de limpiar la sala, recoger la basura:

"Sí, mira se trabaja constantemente aquí, si tú ves... una escuela bien limpia, una escuela que se valora, en el sentido de que si hay papeles en el suelo se les pide o uno mismo quizás recoge toda la basura que hay, y aquí hay una actividad relevante que se hace todos los años que es la caminata ecológica que se relaciona obviamente con el medio ambiente y todo, los niños van disfrazados con animalitos, con basureros llegan todos y se sale a recorrer toda la población". Profesor/a 7.

En relación a lo mismo, el siguiente docente hace una relación exclusiva con la limpieza:

"Bueno, dentro de mi clase siempre está presente el medio ambiente, siempre está el cuidado, el botar la basura donde corresponde, incluso el medio ambiente no solamente lo 
vemos en la parte de limpieza concreta, sino de limpieza de la parte de nuestro lenguaje, de cómo hablamos, de cómo nos referimos a los demás (...)". Profesor/a 8.

Vale mencionar, que en muchas de las ocasiones los profesores/as relacionan directamente las nociones de "cuidado, reciclaje y limpieza", identificándose el uso sinonímico de Medio Ambiente y naturaleza.

\section{Medio Ambiente como Entorno}

Una noción distinta a las anteriores es aquella que asume el Medio Ambiente como "entorno" en el cual habitan los humanos. En grupo de profesores/as que se refieren a Medio Ambiente como lo "que nos rodea":

"Para mí es como el entorno, lo que te rodea y que puedes hacer uso de él y también puedes entregar hacia él, porque no solo lo que te rodea, tú decías denante [sic], tenía que ver con los arbolitos en general, sino también las personas, lo que lo hacemos, lo que lo acompaña, como lo cuidamos, como lo tenemos". Profesor/a 2.

"El medio ambiente es todo lo que nos rodea y que busca que nosotros lo hagamos más sociable también, más amigable hacia nosotros mismos". Profesor/a 22.

“(...) para mí en definitiva, el medio ambiente es todo el espacio que pueda rodear a un ser humano, independiente si es... como te dije antes, urbano, rural, si es mi pieza, mi ciudad, si es mi planeta, pero es todo el espacio en donde yo me desenvuelvo en definitiva, un ser humano". Profesor/a 52.

Se logra identificar que los/as profesores/as reconocen una relación entre el humano y su entorno, vinculándola a su noción de medio ambiente. Es importante mencionar que estos discursos, en contraste a los descritos en el primer apartado, complejizan un poco más las significaciones que se tienen del concepto.

\section{Medio Ambiente como la preocupación por situaciones críticas de degradación del entorno que se habita}

Una tercera noción de Medio Ambiente relaciona el concepto con la descripción de situaciones críticas de degradación del contexto que habitan. De este modo, acentuaron las problemáticas ambientales que se desarrollan en su localidad y definieron Medio Ambiente desde una realidad de conflicto:

"(...) la conciencia de que nosotros también estamos contaminados y es por un tema que lamentablemente nosotros, nuestra zona es de sacrificio. Yo vivo al frente de las termoeléctricas, y ellos [refiriéndose a los estudiantes] en el fondo igual ven el tema como en la medida que ellos puedan contribuir, o sea, la contaminación está, pero lo ven más que nada desde el punto de vista de la sustentabilidad, que era lo que hablamos, que es un 
concepto sobre todo con los más grandes, porque de qué manera yo aporto para que las futuras generaciones en el fondo tengan, no sé, un aire más limpio (...)". Profesor/a 49.

"El tema ambiental es un tema que en el colegio que me desempeño ha cobrado importancia el último tiempo, en gran parte debido al contexto local que vivimos, las grandes mineras, también la minería a mediana escala, también la sequía, la degradación del suelo por uso caprino, son temas que afectan nuestra comuna". Profesor/a 5.

"(...) se nota altiro (sic) en la nariz y la garganta, como duele o como pica, entonces, bueno nosotros tuvimos que también tocar el tema con los niños [haciendo alusión al aire](...)". Profesor/a 44.

En relación a lo anterior, un grupo de profesores construyó además vínculos entre el actual modelo económico y su participación en la crisis ambiental que como sociedad enfrentamos:

“(...) hay mucho problema con el agua, o sea pucha, ahora hay una construcción de un pozo que acá se quiere hacer por el asunto del agua. $Y$ a veces con los niños apuntamos hacia dónde va esto y claramente apunta hacia la minería y desde allí toda la contaminación que ellos producen, todo lo que nos llevan para afuera y después nos lo venden más caro, entonces ahí hay todo un asunto de capitalismo también y claro obviamente con ellos se puede ir conversando y se va mucho más allá que de la simple contaminación del agua o la escasez del agua que puede haber". Profesor/a 2.

En tal sentido, el concepto Medio Ambiente demostró en estos casos el significado de crisis y también de las causas económicas, políticas y culturales de tal crisis ambiental. Este último grupo de profesores relaciona directamente el sistema de relaciones humanas con el concepto, rechazando las nociones a-históricas y a-humanas de Medio Ambiente y el contenido sinonímico de naturaleza que otros profesores han elaborado.

\section{Medio Ambiente y su relación con la disciplina geográfica: desde las Ciencias Naturales al vínculo con lo físico de la Geografía}

Respecto a sí las temáticas medioambientales eran trabajadas dentro de la Geografía, un grupo mayor de profesores/as señaló que éstas eran trabajadas en el marco de las Ciencias Naturales y no en los contenidos de Geografía de la asignatura Historia, Geografía y Ciencias Sociales, indicando muchas veces que estaba establecido así en las bases curriculares:

"Yo creo que los contenidos de Geografía apoyan en este caso [refiriéndose a Medio Ambiente] a lo de Ciencias Naturales. Yo por lo general lo paso más [haciendo alusión al concepto Medio Ambiente] en Ciencias Naturales que, en Historia, que en Geografía". Profesor/a 12.

“(...) el tema medioambiental no viene tratado propiamente tal en esto [refiriéndose a la Geografía], sino más bien en los módulos de Ciencias (...) todo lo que es hábitat, de hecho, 
estamos hablando de lo que es ecosistemas, biomas y eso tiene relación con todo el tema ambiental". Profesor/a 21.

Uno de los profesores, además, explicita que desde su perspectiva el trabajo con temáticas ambientales tenía más relación con Ciencias Naturales que con Geografía:

"Con la naturaleza, porque viene de la naturaleza también, viene en ciencias naturales ese tema, pero según lo que yo creo puede ser más enfocado [el Medio Ambiente] desde la naturaleza que desde la geografía". Profesor/a 37.

En estas palabras se vuelve a reconocer el fuerte vínculo que une sus concepciones de Medio Ambiente con el concepto de naturaleza, muchas veces teniendo un trato sinonímico, en el cual las relaciones construidas por los humanos no tienen tanta cabida en la construcción de un Medio Ambiente en comparación a los ecosistemas o biomas.

No obstante, a esto, un reducido grupo de profesores explicó que consideraban que tales materias debieran ser abordadas desde la Geografía; sin embargo, en su mayoría construyen una relación exclusiva con la arista física de la Geografía:

“(...) tiene relación sin duda, medio ambiente... los climas están determinados por la zona geográfica, que son... eh claro, absoluta relación si, de esa manera. Lo que hablábamos denante [sic] del altiplano o clima mediterráneo, ahí hay una relación". Profesor/a 21.

“(...) yo creo que está muy ligado la Geografía. (...) Por ejemplo una vez que salimos a recorrer el valle de Elqui, quedaron los niños impresionadísimos con esos cerros tan altos, incluso hicieron historias sobre esos cerros tan altos y el valle tan angosto, tanto que produce y la diversidad que tiene, eso fue muy significativo para mí como profesora y para los niños, igual la visita al bosque Fray Jorge, también, ver este mundo verde de llovizna, de neblina y un poco más abajo todo árido, todo seco". Profesor/a 36.

Por ende, queda evidenciado que no todos los profesores logran construir un vínculo sólido entre el concepto Medio Ambiente y la Educación Geográfica, quienes logran hacerlo, sólo lo hacen con una perspectiva física de la Geografía, carente de reflexión del papel de la sociedad humana, ausente de análisis de las relaciones internas entre los grupos humanos y cómo éstas influyen en las condiciones ambientales que actualmente enfrentamos.

\section{Conclusión: Asimetría entre los elementos conceptuales y axiológicos de los significados de Medio Ambiente}

Para comprender los significados que estos docentes de primaria tienen sobre el Medio Ambiente es necesario distinguir entre los elementos conceptuales y los axiológicos. Ambos tipos de elementos forman parte fundamental del contenido de los significados que tienen los/as profesores/as sobre el Medio Ambiente, por ende, desde la perspectiva que lo enseñan.

Los elementos conceptuales se relacionan con la comprensión disciplinaria, científica y académica de Medio Ambiente. Estos elementos conceptuales son bastante confusos en los signifi- 
cados de estos profesores. Por ejemplo, al identificar casi como sinónimos a Medio Ambiente con Naturaleza y al Entorno con los elementos físicos. Es decir, Naturaleza y elementos físicos tienen una prevalencia, un poder explicativo mayor y más arraigado que Medio Ambiente.

Por otra parte, los elementos axiológicos son más nítidos y más elaborados que los conceptuales: el Medio Ambiente tiene existencia real, está en peligro, depende de nosotros y se propicia un cambio conductual a través de acciones de protección y cuidado. En determinados casos esos cambios conductuales tienen el carácter de colectivos y culturales. Sin embargo, en la mayoría de ellos, los significados se sitúan más bien en un objeto de cuidado que en un sujeto de relaciones humanos-espaciales.

Por lo tanto, se constata una asimetría entre los elementos conceptuales y axiológicos de los significados de Medio Ambiente que tiene estos profesores/as chilenos: hay un alto valor y claridad por la necesidad de proteger y desarrollar acciones de protección, incluso, parte de nuestra vida presente como individuos y sociedad, depende de ello. Esto, aunque no se identifica con claridad a nivel teórico, qué es lo que hay que proteger y cuidar: la especificidad del Medio Ambiente.

\section{Referencias bibliográficas}

ALIMONDA, H. La naturaleza colonizada. Buenos Aires: CICCUS, 2011.

ÂNGELO, S. Natureza e ambiente no ensino da geografia. In: DE SOUZA; L. APARECIDA, M. \& CAMILO, V. Produção do conhecimento e pesquisa no ensino da geografia. Goiânia: Editora da PUC Goiás, 2011, p. 139-148.

ARAYA, F. (editor). Formación Ciudadana desde la Educación Geográfica. La Serena: Editorial Universidad de La Serena.

ARENAS, A. y SALINAS, V. Giros en la educación geográfica basados en los enfoques renovados de lo geográfico y lo educativo. Panorama a partir de una revisión bibliográfica. Revista de Geografía Norte Grande, 2013, N 56, p. 143-162.

ARENAS-MARTIJA, A.; SALINAS-SILVA, S.; MARGALEF-GARCÍA, L. \& OTERO-AURISTONDO, M. Fragility of Pedagogical Content Knowledge in Geography. Journal of Geography, 2016, Vol. 116, p. 57-66.

ARENAS, A.; FERNÁNDEZ, H. y PÉREZ, P. (editores). Una Educación Geográfica para Chile. Santiago de Chile: Sociedad Chilena de Ciencias Geográficas, 2016. Disponible en internet: http://sociedadchilenadecienciasgeograficas.cl/2014/wp-content/uploads/2016/11/SOCHIGEO-2016-UNA-EDUCACION-GEOGRAFICA-PARA-CHILE.pdf

ARENAS-MARTIJA, A.; PERÉZ-GALLARDO, P.; SALINAS-SILVA, S. \& OTERO-AURISTONDO, M. What Type of Geography Do We Teach? from Theoretical-Conceptual Weaknesses to Underestimation of Spatial Experience. Chilean Teachers' Views on Teaching Geography. In: BROOKS, C.; BUTT, G.; \& FARGHER, M. (editors). The Power of Geographical Thinking. Londres: Springer, 2017, p. 75-90. 
ÁVALOS, B. (1999). Desarrollo docente en el contexto de la institución escolar. Los microcentros rurales y los grupos profesionales de trabajo en Chile. En: Ponencia Conferencia Los Maestros en América Latina: Nuevas Perspectivas sobre su Desarrollo y Desempeño. San José, Costa Rica, 2830 junio de 1999. Disponible en Internet:

https://publications.iadb.org/handle/11319/2394?scope=123456789/12\&thumbnail=false\&order=desc\&rpp $=5 \&$ sort_by=score \&page $=1 \& q u e r y=E I+R o l+d e+l o s+B a n c o s+N a c i o n a l e s+d e+$ Desarrollo+ catalizar+el+financiamiento+clim\%25C3\%25A1tico+internacional\&group_by=none\&etal=0\&filtertype_O=country_en\&filter_O=Chile\&filter_relational_operator_O=equals. 10/03/2013.

BERGER, P. y LUCKMANN, T. La construcción social de la realidad. Buenos Aires: Amorrortu, 1968.

BIANCHI, R. (compiladora). Un incentivo para el conocimiento geográfico y la resolución de problemas espaciales destinado a profesores de la enseñanza básica/primaria en países latinoamericanos. Santiago de Chile: LOM, Financiado por el Instituto Panamericano de Geografía e Historia (IPGH-OEA), 2017.

CALLAI, H. \& DE MORAES, M.M. Pesquisa, Educacao e Ciudadania. Percursos Teóricos e Metodológicos. Injuí: Brasil, Editorial Unijuí, 2016.

CARDOSO, V. A prática dos professores de geografia: um diagnóstico da educação ambiental no ensino médio do estado de Goiás. In: DA SILVA, \& E. MENDES, L. (editores). Desafios da Didática de Geografía. Goiânia: Editora da PUC Goiás, 2013, p. 195-213.

CASTORIADIS, C. Institución imaginaria de la sociedad. Ciudad de México: Tuesquets Editores, 2013.

CAVALCANTI, L. A Educação geográfica e a formação de conceitos: a importância do lugar na ensino de geografia. En: GARRIDO, M. (editor). La Espesura del Lugar. Reflexiones sobre el espacio en el mundo educativo. Santiago de Chile: Universidad Academia de Humanismo Cristiano, 2009, p. 135-152.

DENZIN, N. \& LINCOLN, Y. The SAGE handbook of qualitative research. London: SAGE, 2005.

FLICK, U. Introducción a la Investigación Cualitativa. Madrid: Morata, 2004.

FLICK, U. El diseño de la Investigación Cualitativa. Madrid: Morata, 2014.

GALLASTEGUI, J. \& GALEA, J. Espacios para una geografía social, humanista y crítica. Valparaíso: Universidad de Playa Ancha, 2009.

GARRIDO, M. El espacio por aprender, el mismo que enseñar: las urgencias de la educación geográfica. Cad. Cedes, 2005, Vol. 25, № 66, p. 137-163.

GIBBS, G. El análisis de datos cualitativos en Investigación Cualitativa. Madrid: Morata, 2012.

GOUROU, P. Introducción a la geografía humana. Madrid: Alianza Editorial, 1973. 
GREGORY, D.; JOHNSTON, R.; PRATT, G.; WATTS, M. \& WHATMORE, S. The dictionary of Human Geography. Sussex: Wiley-Blackwell, 2009.

GUDYNAS. E. La ecología política de la integración: reconstrucción de la ciudadanía y regionalismo autónomo. En: ALIMONDA, E. Ecología política, naturaleza, sociedad y utopía. Buenos Aires: CLACSO, 2002, p. 137-152.

GUDYNAS, E. Diez tesis urgentes sobre el nuevo extractivismo. En: Extractivismo, política y sociedad. Quito: CLAES, 2009, p. 187-225.

GUREVICH, R. Ambiente y Educación. Una apuesta al futuro. Buenos Aires: Editorial Paidós, 2011.

HARAWAY, D. Anthropocene, Capitalocene, Plantationocene, Chthulucene: Making Kin. Environmental Humanities, 2015, Vol. 6, p.159-165.

HARVEY, D. A brief history of neoliberalism. New York: Oxford University Press, 2007.

HINCHLIFFE, S. Nature/Culture. In: ATKINSON, D.; JACKSON, P.; SIBLEY, D. \& WASHBOURNE, N. Cultural Geography, a critical dictionary of key concepts. London: I.B. Tauris \& Co. Ltd., 2005, p. 194-199.

LEFF, E. Saber ambiental, sustentabilidad, racionalidad, complejidad, poder. Ciudad de México: Siglo XXI Editores, 2007.

LEFF, E. Discursos Sustentables. México: Siglo XXI Editores, 2008.

LITTLE, J. Nature, Fear and Rurality. In: PAIN, R. \& SMITH, S. Fear: Critical Geopolitics and Everyday Life. Hampshire: Ashgate Publishing Limited, 2008, p. 87-102.

LONGHURST, R. Semi-structured interviews and Focus Groups. In: CLIFFORD, N.; FRENCH, S. \& VALENTINE. G. Key Methods in Geography. London: SAGE Publications Ltd, 2010, p. 103-115.

MASSEY, D. Lugar, identidad y geografías de la responsabilidad en un mundo en proceso de globalización. Revista Treballs de la Societat Catalana de Geografía, 2004, N 57, p. 77-84.

MCDOWELL, L. Género, Identidad y Lugar. Madrid: Ediciones Cátedra, 2000.

MINISTERIO DE EDUCACIÓN DE CHILE (MINEDUC). Historia, Geografía y Ciencias Sociales, Cuaderno de Trabajo, Geografía. Módulo didáctico para la enseñanza y el aprendizaje en escuelas rurales multigrado. Geografía I, Clase 1. 10 a 60 Básico. Santiago de Chile: MINEDUC, 2014. Disponible en Internet: https://rural.mineduc.cl/modulos-multigrado-historia-geografia-ciencias-sociales/

MOREIRA, R. Geografia e práxis. São Paulo: Contexto, 2012.

NOGUÉ, J. y ROMERO, J. Otras geografías, otros tiempos. Nuevas y viejas preguntas, viejas y nuevas respuestas. En: NOGUÉ, J. y ROMERO, J. (editores). Las otras Geografías. Valencia: Tirant lo Blanch, 2006, p. 15-50. 
NOGUÉ, J. (editor). La construcción social del paisaje. Madrid: Editorial Biblioteca Nueva España, 2007.

PORTO-GONÇALVES, C. GEO-GRAFÍAS. Movimientos sociales, nuevas territorialidades y sustentabilidad. Ciudad de México: Siglo XXI, 2001.

SANTOS, M. Técnica, Espaço, Tempo. São Paulo: EDUSP, 2004.

SARIBAS, D.; DOGANCA, Z. \& ERTEPINAR, H. Implementation of an environmental education course to improve pre-service elementary teachers' environmental literacy and self-efficacy beliefs. International Research in Geographical and Environmental Education, 2017, Vol. 26, № 4, p. 311-326.

SCHWANDT, T. Constructivist, interpretivist approaches to human inquiry. In: DENZIN, N. \& LINCOLN, Y. (editors). Handbook of qualitative research. London: Sage, 1998, p. 118-137.

SMITH, N. El rendimiento de las ciudades: la globalización y el urbanismo neoliberal. En: HARVEY, D. \& SMITH, N. Capital financiero, propiedad inmobiliaria y cultura. Barcelona: Universidad Autónoma de Barcelona, 2005, p. 59-78.

SOJA, E. Postmetrópolis, estudios críticos sobre las ciudades y las regiones. Madrid: Ed. Traficantes de sueños, 2008.

TUAN, Y.F. Topofilia: un estudio de las percepciones, actitudes y valores sobre el entorno. Santa Cruz de Tenerife: Melusina, 2007.

VAN DOREEN, T. Life and Loss at the Edge of Extinction. New York: Columbia University Press, 2014. 